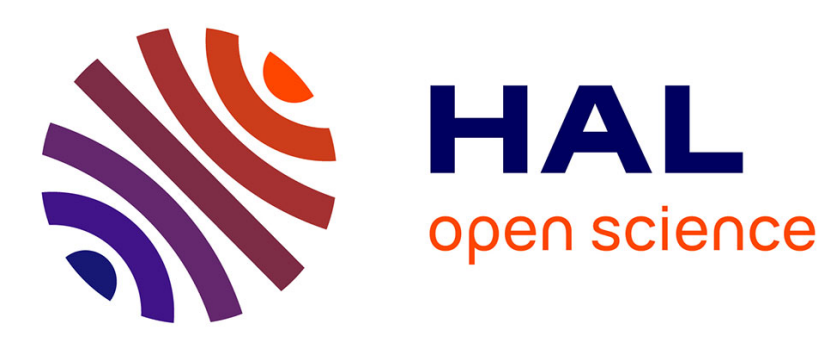

\title{
Equivalence of local-best and global-best approximations in $\mathbf{H}($ curl)
}

Théophile Chaumont-Frelet, Martin Vohralík

\section{To cite this version:}

Théophile Chaumont-Frelet, Martin Vohralík. Equivalence of local-best and global-best approximations in $\mathrm{H}$ (curl). Calcolo, 2021, 58, pp.53. 10.1007/s10092-021-00430-9 . hal-02736200v2

\section{HAL Id: hal-02736200 \\ https://hal.inria.fr/hal-02736200v2}

Submitted on 27 Sep 2021

HAL is a multi-disciplinary open access archive for the deposit and dissemination of scientific research documents, whether they are published or not. The documents may come from teaching and research institutions in France or abroad, or from public or private research centers.
L'archive ouverte pluridisciplinaire HAL, est destinée au dépôt et à la diffusion de documents scientifiques de niveau recherche, publiés ou non, émanant des établissements d'enseignement et de recherche français ou étrangers, des laboratoires publics ou privés. 


\title{
Equivalence of local-best and global-best approximations in $\boldsymbol{H}(\mathrm{curl})^{*}$
}

\author{
Théophile Chaumont-Frelet ${ }^{\dagger \ddagger} \quad$ Martin Vohralík $\mathbf{I}^{\S}$
}

September 27, 2021

\begin{abstract}
We derive results on equivalence of piecewise polynomial approximations of a given function in the Sobolev space $\boldsymbol{H}$ (curl). We namely show that the global-best approximation of a given $\boldsymbol{H}$ (curl) function in a $\boldsymbol{H}$ (curl)-conforming piecewise polynomial space imposing the continuity of the tangential trace can be bounded above and below by the Hilbertian sum of the respective local approximations from the elementwise spaces without any inter-element continuity requirement. In other words, the approximation of a $\boldsymbol{H}$ (curl) function by tangential-trace-continuous and discontinuous piecewise polynomials has comparable precision. We consider approximations of the curl of the target function in the $\boldsymbol{L}^{2}$-norm, as well as approximations of the target function in the $\boldsymbol{L}^{2}$-norm with a constraint on the curl; in the latter case, the constraint is removed in the local approximations. These best-approximation localizations hold under the minimal $\boldsymbol{H}$ (curl) regularity, on arbitrary shape-regular tetrahedral meshes, and involve imposition of conditions on a part of the boundary. They extend to the $\boldsymbol{H}$ (curl) context some recent results from the $H^{1}$ and $\boldsymbol{H}$ (div) spaces and have direct applications to a priori and a posteriori error analysis of numerical discretizations related to the $\boldsymbol{H}$ (curl) space, namely Maxwell's equations.
\end{abstract}

Key words: Sobolev space $\boldsymbol{H}$ (curl), tangential trace continuity, minimal regularity, constrained approximation, unconstrained approximation, a priori error estimate, a posteriori error estimate, finite element method, edge elements

\section{Introduction}

Let $\Omega \subset \mathbb{R}^{3}$ be an open, Lipschitz, simply-connected polyhedron. Let $v \in H^{1}(\Omega)$ be given, together with a polynomial degree $p \geq 1$ and a tetrahedral mesh of $\Omega$ (see below in Section 2 for the details of the notation and setting we use). Then the error in the approximation of the function $v$ by trace-continuous $\left(H^{1}(\Omega)\right.$ conforming) piecewise $p$-degree polynomials is up to a generic constant equivalent to the approximation of $v$ by discontinuous (no inter-element continuity required) piecewise $p$-degree polynomials. This result was first formulated by Veeser [35], extending some earlier contributions by Carstensen et al. [9, Theorem 2.1 and inequalities (3.2), (3.5), and (3.6)] and Aurada et al. [3, Proposition 3.1]. Bank and Yserentant [5] have obtained a similar result where, however, the target function has to be smoother than $H^{1}(\Omega)$ and a sufficiently refined mesh is needed. Further extensions are presented in Veeser [36] and Tantardini et al. [34], whereas an improvement of the dependence of the equivalence constant on the polynomial degree in two space dimensions (from algebraic to logarithmic) is developed in [8, Theorem 4]. We recall this result in details in Proposition 3.1 below.

Similarly, let $\boldsymbol{v} \in \boldsymbol{H}(\operatorname{div}, \Omega)$ be given. Then the error in the approximation of the function $\boldsymbol{v}$ by normaltrace-continuous $(\boldsymbol{H}(\operatorname{div}, \Omega)$-conforming) piecewise polynomials with the divergence fixed by $\nabla \cdot \boldsymbol{v}$ is up to a generic constant equivalent to the approximation of $\boldsymbol{v}$ by discontinuous (no inter-element continuity required)

\footnotetext{
*This project has received funding from the European Research Council (ERC) under the European Union's Horizon 2020 research and innovation program (grant agreement No 647134 GATIPOR).

${ }^{\dagger}$ Inria, 2004 Route des Lucioles, 06902 Valbonne, France (theophile.chaumont@inria.fr).

$\ddagger$ Laboratoire J.A. Dieudonné, Parc Valrose, 28 Avenue Valrose, 06108 Nice, France

$\S$ Inria, 2 rue Simone Iff, 75589 Paris, France (martin.vohralik@inria.fr).

I CERMICS, Ecole des Ponts, 77455 Marne-la-Vallée, France
} 
piecewise polynomials without any constraint on the divergence. This result was recently obtained in Ern et al. [19], in the philosophy of the above $H^{1}(\Omega)$-result, cf. also [10] for some early ideas on the removal of the divergence constraint. In Bank and Ovall [4], again a sufficiently smooth target function and a sufficiently refined mesh are needed for a similar result. We recall this result in details in Proposition 3.2 below.

The two results above are fundamental with numerous direct applications to a priori and a posteriori error analysis related to the $H^{1}(\Omega)$ and $\boldsymbol{H}(\operatorname{div}, \Omega)$ spaces. To the best of our knowledge, such a result is not available for the Sobolev space $\boldsymbol{H}(\operatorname{curl}, \Omega)$, and it is our purpose to obtain it here. We actually show that it can be easily derived from the two above ones. Indeed, in addition to Propositions 3.1 and 3.2, we will merely use the fundamental properties that 1) each function from the $H^{1}(\Omega)$ space whose weak gradient vanishes is a real constant; 2) each function from the $\boldsymbol{H}(\operatorname{curl}, \Omega)$ space whose weak curl vanishes is a gradient of a function from $H^{1}(\Omega)$; and 3) each function from the $\boldsymbol{H}(\operatorname{div}, \Omega)$ space whose weak divergence vanishes is a curl of a function from $\boldsymbol{H}(\operatorname{curl}, \Omega)$, which is expressed in the exact sequence

$$
\mathbb{R} \hookrightarrow H^{1}(\Omega) \stackrel{\nabla}{\longrightarrow} \boldsymbol{H}(\operatorname{curl}, \Omega) \stackrel{\nabla \times}{\longrightarrow} \boldsymbol{H}(\operatorname{div}, \Omega) \stackrel{\nabla \cdot}{\longrightarrow} L^{2}(\Omega),
$$

see, e.g., Arnold et al. [2] and the references therein; we will also consider discrete versions of (1.1) and imposition of conditions on a part of the boundary in (1.1), which can be accommodated following Girault and Raviart [25], Fernandes and Gilardi [24], and the references therein.

The question of equivalence of local-best and global-best approximations is related to the design of (quasi)interpolation operators. The canonical interpolation operator in $\boldsymbol{H}$ (curl, $\Omega$ ) of [7, 29] maps any function that is sufficiently smoother than $\boldsymbol{H}(\operatorname{curl}, \Omega)$ to a piecewise polynomial, finite-dimensional Nédélec [29] subspace of $\boldsymbol{H}$ (curl, $\Omega$ ) via a prescription of degrees of freedom. The canonical interpolation operator is heavily used but its deficiency is that it cannot be applied to a function only having the minimal $\boldsymbol{H}(\operatorname{curl}, \Omega)$ regularity. This can, for instance, be overcome via mollification, see Schöberl [31, 32], Christiansen and Winther [13], Falk and Winther [23], Ern and Guermond [20], or Licht [26]; employing integral moments in the spirit of the Clément [15] or Scott-Zhang [33] interpolation operator, cf. Bernardi and Hecht [6]; by splitting of the target function and its curl into a regular part and a gradient as in Ciarlet [14]; or employing local projections and averaging as in Ern and Guermond [21]. A special care has to be taken if approximation properties both in the mesh size $h$ and the polynomial degree $p$ are important, see, e.g., Demkowicz and Buffa [18], Demkowicz [17], and most recently Melenk and Rojik [27] and Ern et al. [19]; we do not address this point here. One of the main uses of (quasi-)interpolation operators is to derive optimal a priori error estimates in numerical approximations; for the application of the above results in the context of Maxwell's equations (cf. Monk [28]) under low regularity, we refer to Ciarlet [14], Ern and Guermond [22], ChaumontFrelet et al. [12], and the references therein. Our main results, Theorems 4.1 and 4.2, can immediately be used for this purpose, and this under the minimal $\boldsymbol{H}$ (curl, $\Omega$ ) regularity, on arbitrary shape-regular tetrahedral meshes, and for essential conditions only imposed on a part of the boundary. More generally, our results can serve as building blocks for developments of minimal-regularity quasi-interpolation operators in a priori error analysis, and they also provide sharper and simpler characterization of equivalence classes used in convergence and optimality proofs of finite element schemes using a-posteriori-steered mesh refinement, cf. the discussion in [35] and the references therein.

This contribution is organised as follows. In Section 2, we introduce the continuous- and discrete-level spaces we work with. In Section 3, we recall the known $H^{1}(\Omega)$ and $\boldsymbol{H}(\operatorname{div}, \Omega)$ local-global equivalences from [35] and [19], respectively. Section 4 introduces our two main results, the equivalences of globaland local-best approximations in $\boldsymbol{H}(\operatorname{curl}, \Omega)$, in the $\boldsymbol{H}(\operatorname{curl}, \Omega)$-seminorm without any constraint and in the $\boldsymbol{L}^{2}(\Omega)$-norm under the constraint on the curl, which is removed in the local-best approximation. Finally, Appendix A extends an important technical result on constrained-unconstrained equivalence on a tetrahedron of [19, Lemma A.1], based on Costabel and McIntosh [16, Proposition 4.2], to the $\boldsymbol{H}(\operatorname{curl}, \Omega)$ setting.

\section{Setting and notation}

This section introduces continuous- and discrete-level functional spaces we will be working with.

\subsection{Basic continuous-level spaces}

We denote by $L^{2}(\Omega)$ the space of square-integrable functions defined on $\Omega$ and we set $\boldsymbol{L}^{2}(\Omega):=\left[L^{2}(\Omega)\right]^{3}$. $H^{1}(\Omega)$ is the space of scalar-valued $L^{2}(\Omega)$ functions with weak gradients in $\boldsymbol{L}^{2}(\Omega), H^{1}(\Omega):=\{v \in$ 
$\left.L^{2}(\Omega) ; \nabla v \in \boldsymbol{L}^{2}(\Omega)\right\}$. We refer the reader to [1] for an in-depth description of these spaces. Next, $\boldsymbol{H}(\operatorname{curl}, \Omega)$ is the space of vector-valued $\boldsymbol{L}^{2}(\Omega)$ functions with weak curls in $\boldsymbol{L}^{2}(\Omega), \boldsymbol{H}(\operatorname{curl}, \Omega):=\left\{\boldsymbol{v} \in \boldsymbol{L}^{2}(\Omega) ; \nabla \times \boldsymbol{v} \in\right.$ $\left.\boldsymbol{L}^{2}(\Omega)\right\}$. We will also need $\boldsymbol{H}(\operatorname{div}, \Omega)$, the space of vector-valued $\boldsymbol{L}^{2}(\Omega)$ functions with weak divergences in $L^{2}(\Omega), \boldsymbol{H}(\operatorname{div}, \Omega):=\left\{\boldsymbol{v} \in \boldsymbol{L}^{2}(\Omega) ; \nabla \cdot \boldsymbol{v} \in L^{2}(\Omega)\right\}$. Finally, let $\boldsymbol{H}^{1}(\Omega):=\left\{\boldsymbol{v} \in \boldsymbol{L}^{2}(\Omega) ; \boldsymbol{v}_{i} \in H^{1}(\Omega), i=\right.$ $1, \ldots, 3\}$. The reader will find a complete description of these vector-valued Sobolev spaces in [25]. Below, we denote by $\|\cdot\|_{\omega}$ the $L^{2}(\omega)$ or $\boldsymbol{L}^{2}(\omega)$ norm and by $(\cdot, \cdot)_{\omega}$ the associated scalar product; we drop the index when $\omega=\Omega$.

\subsection{Continuous-level spaces with conditions on a part of the boundary}

In the sequel, we will allow for prescribing boundary conditions on part of the boundary $\partial \Omega$. Let $\Gamma_{\mathrm{D}}$, $\Gamma_{\mathrm{N}}$ be two disjoint open, possibly empty, subsets of $\partial \Omega$ such that $\partial \Omega=\overline{\Gamma_{\mathrm{D}}} \cup \overline{\Gamma_{\mathrm{N}}}$ and such that $\Gamma_{\mathrm{D}}$ is connected; moreover, each boundary face of the mesh defined below is requested to lie entirely either in $\overline{\Gamma_{\mathrm{D}}}$ or in $\overline{\Gamma_{\mathrm{N}}}$. Then $H_{0, \mathrm{D}}^{1}(\Omega)$ is the subspace of $H^{1}(\Omega)$ formed by functions vanishing on $\Gamma_{\mathrm{D}}$ in the sense of traces, $H_{0, \mathrm{D}}^{1}(\Omega):=\left\{v \in H^{1}(\Omega) ; v=0\right.$ on $\left.\Gamma_{\mathrm{D}}\right\}$. In particular, $H_{0, \mathrm{D}}^{1}(\Omega)=H^{1}(\Omega)$ when $\Gamma_{\mathrm{D}}=\emptyset$. Let $\mathrm{T}=\mathrm{D}$ or $\mathrm{N}$; then $\boldsymbol{H}_{0, \mathrm{~T}}(\operatorname{curl}, \Omega)$ is the subspace of $\boldsymbol{H}(\operatorname{curl}, \Omega)$ formed by functions with vanishing tangential trace on $\Gamma_{\mathrm{T}}, \boldsymbol{H}_{0, \mathrm{~T}}(\operatorname{curl}, \Omega):=\left\{\boldsymbol{v} \in \boldsymbol{H}(\operatorname{curl}, \Omega) ; \boldsymbol{v} \times \boldsymbol{n}=0\right.$ on $\left.\Gamma_{\mathrm{T}}\right\}$, where $\boldsymbol{v} \times \boldsymbol{n}=0$ on $\Gamma_{\mathrm{T}}$ means that $(\nabla \times \boldsymbol{v}, \boldsymbol{\varphi})-(\boldsymbol{v}, \nabla \times \boldsymbol{\varphi})=0$ for all functions $\boldsymbol{\varphi} \in \boldsymbol{H}^{1}(\Omega)$ such that $\boldsymbol{\varphi} \times \boldsymbol{n}=\mathbf{0}$ on $\partial \Omega \backslash \Gamma_{\mathrm{T}}$. Finally, $\boldsymbol{H}_{0, \mathrm{~N}}(\operatorname{div}, \Omega)$ is the subspace of $\boldsymbol{H}(\operatorname{div}, \Omega)$ formed by functions with vanishing normal trace on $\Gamma_{\mathrm{N}}$, $\boldsymbol{H}_{0, \mathrm{~N}}(\operatorname{div}, \Omega):=\left\{\boldsymbol{v} \in \boldsymbol{H}(\operatorname{div}, \Omega) ; \boldsymbol{v} \cdot \boldsymbol{n}=0\right.$ on $\left.\Gamma_{\mathrm{N}}\right\}$, where $\boldsymbol{v} \cdot \boldsymbol{n}=0$ on $\Gamma_{\mathrm{N}}$ means that $(\boldsymbol{v}, \nabla \varphi)+(\nabla \cdot \boldsymbol{v}, \varphi)=0$ for all functions $\varphi \in H_{0, \mathrm{D}}^{1}(\Omega)\left(\boldsymbol{H}_{0, \mathrm{D}}(\operatorname{div}, \Omega)\right.$ will also occasionally be used). We refer the reader to [24] for a thorough characterization of tangential (respectively normal) traces of $\boldsymbol{H}(\operatorname{curl}, \Omega)($ resp. $\boldsymbol{H}(\operatorname{div}, \Omega)$ ) on a part of the boundary.

\subsection{Tetrahedral mesh}

Let $\mathcal{T}_{h}$ be a simplicial mesh of the domain $\Omega$, i.e., $\cup_{K \in \mathcal{T}_{h}} K=\bar{\Omega}$, any element $K \in \mathcal{T}_{h}$ is a tetrahedron with nonzero measure, and the intersection of two different tetrahedra is either empty, a vertex, an edge, or a face; all these geometrical objects are supposed to be closed. As mentioned above, we assume that each boundary mesh face is entirely contained either in $\overline{\Gamma_{\mathrm{D}}}$ or in $\overline{\Gamma_{\mathrm{N}}}$. The shape-regularity parameter of the mesh $\mathcal{T}_{h}$ is the positive real number

$$
\kappa_{\mathcal{T}_{h}}:=\max _{K \in \mathcal{T}_{h}} \frac{h_{K}}{\rho_{K}},
$$

where $h_{K}$ is the diameter of the tetrahedron $K$ and $\rho_{K}$ is the diameter of the largest ball contained in $K$.

\subsection{Discrete-level spaces}

Let an integer $p \geq 0$ and a tetrahedron $K \in \mathcal{T}_{h}$ be fixed. We denote by $\mathcal{P}_{p}(K)$ the space of scalar-valued polynomials on $K$ of total degree at most $p$ and by $\left[\mathcal{P}_{p}(K)\right]^{3}$ is the space of vector-valued polynomials on $K$ whose each component lies in $\mathcal{P}_{p}(K)$. The Nédélec [7,29] space of degree $p$ on $K$ is then given by

$$
\mathcal{N}_{p}(K):=\left[\mathcal{P}_{p}(K)\right]^{3}+\boldsymbol{x} \times\left[\mathcal{P}_{p}(K)\right]^{3} .
$$

Similarly, the Raviart-Thomas $[7,30]$ space of degree $p$ on $K$ is given by

$$
\mathcal{R T}_{p}(K):=\left[\mathcal{P}_{p}(K)\right]^{3}+\mathcal{P}_{p}(K) \boldsymbol{x} ;
$$

we note that (2.1) and (2.2) are equivalent to the writing with a direct sum and only homogenous polynomials in the second term. We will extensively use below the broken, piecewise polynomial spaces formed from these element spaces

$$
\begin{aligned}
\mathcal{P}_{p}\left(\mathcal{T}_{h}\right) & :=\left\{v_{h} \in L^{2}(\Omega) ;\left.v_{h}\right|_{K} \in \mathcal{P}_{p}(K) \quad \forall K \in \mathcal{T}_{h}\right\}, \\
\mathcal{N}_{p}\left(\mathcal{T}_{h}\right) & :=\left\{\boldsymbol{v}_{h} \in \boldsymbol{L}^{2}(\Omega) ;\left.\boldsymbol{v}_{h}\right|_{K} \in \mathcal{N}_{p}(K) \quad \forall K \in \mathcal{T}_{h}\right\}, \\
\mathcal{R T}_{p}\left(\mathcal{T}_{h}\right) & :=\left\{\boldsymbol{v}_{h} \in \boldsymbol{L}^{2}(\Omega) ;\left.\boldsymbol{v}_{h}\right|_{K} \in \mathcal{R T}_{p}(K) \quad \forall K \in \mathcal{T}_{h}\right\}
\end{aligned}
$$

Finally, to form the usual finite-dimensional subspaces of the spaces of Sections 2.1 and 2.2, we will write $\mathcal{P}_{p}\left(\mathcal{T}_{h}\right) \cap H^{1}(\Omega)$ (for $\left.p \geq 1\right), \mathcal{N}_{p}\left(\mathcal{T}_{h}\right) \cap \boldsymbol{H}(\operatorname{curl}, \Omega), \mathcal{R T}_{p}\left(\mathcal{T}_{h}\right) \cap \boldsymbol{H}(\operatorname{div}, \Omega)$ (for $p \geq 0$ ), and similarly for subspaces reflecting the different boundary conditions. 


\section{Equivalence of local-best and global-best approximations in $H^{1}$ and $\boldsymbol{H}(\operatorname{div})$}

We recall here the known $H^{1}(\Omega)$ - and $\boldsymbol{H}(\operatorname{div}, \Omega)$-local/global equivalences from [35] and [19], respectively. Hereafter, we will use the notation $a \lesssim b$ when there exists a positive real constant $C$ such that $a \leq C b$. Similarly, $a \approx b$ means that $a \lesssim b$ and $b \lesssim a$ simultaneously. The possible dependencies of $C$ will always be explicitly stated.

\subsection{The $H^{1}$ case}

The following result can be easily seen along the lines of [35, Corollaries 1 and 2], where $\Gamma_{D}=\partial \Omega$ or $\Gamma_{D}=\emptyset$ is considered.

Proposition 3.1 (Equivalence of local-best and global-best approximations in $H^{1}, H^{1}$ seminorm). Let $p \geq 1$. Then there exists a constant only depending on the shape-regularity $\kappa_{\mathcal{T}_{h}}$ of the mesh $\mathcal{T}_{h}$ and the polynomial degree $p$ such that for any $v \in H_{0, \mathrm{D}}^{1}(\Omega)$,

$$
\min _{v_{h} \in \mathcal{P}_{p}\left(\mathcal{T}_{h}\right) \cap H_{0, \mathrm{D}}^{1}(\Omega)}\left\|\nabla\left(v-v_{h}\right)\right\|^{2} \approx \sum_{K \in \mathcal{T}_{h}} \min _{v_{h} \in \mathcal{P}_{p}(K)}\left\|\nabla\left(v-v_{h}\right)\right\|_{K}^{2} .
$$

Note that the minimization terms in the right-hand side of (3.1) are completely local in each tetrahedron and do not involve any constraint on the value on faces in $\partial K \cap \overline{\Gamma_{\mathrm{D}}}$. The approximation by continuous piecewise polynomials is thus comparable to that by discontinuous piecewise polynomials whenever the target function has $H^{1}(\Omega)$-regularity.

\subsection{The $\boldsymbol{H}(\operatorname{div})$ case}

The following result is proved in [19, Theorem 3.3]:

Proposition 3.2 (Equivalence of local-best and global-best approximations in $\boldsymbol{H}$ (div), $\boldsymbol{L}^{2}$-norm under the constraint on the divergence (removing the constraint)). Let $p \geq 0$. Then there exists a constant only depending on the shape-regularity $\kappa_{\mathcal{T}_{h}}$ of the mesh $\mathcal{T}_{h}$ and the polynomial degree $p$ such that for any $\boldsymbol{v} \in \boldsymbol{H}_{0, \mathrm{~N}}(\operatorname{div}, \Omega)$ with $\nabla \cdot \boldsymbol{v} \in \mathcal{P}_{p}\left(\mathcal{T}_{h}\right)$,

$$
\min _{\substack{\boldsymbol{v}_{h} \in \mathcal{R} \mathcal{T}_{p}\left(\mathcal{T}_{h}\right) \cap \boldsymbol{H}_{0, \mathrm{~N}}(\operatorname{div}, \Omega) \\ \nabla \cdot \boldsymbol{v}_{h}=\nabla \cdot \boldsymbol{v}}}\left\|\boldsymbol{v}-\boldsymbol{v}_{h}\right\|^{2} \approx \sum_{K \in \mathcal{T}_{h}} \min _{\boldsymbol{v}_{h} \in \mathcal{R} \mathcal{T}_{p}(K)}\left\|\boldsymbol{v}-\boldsymbol{v}_{h}\right\|_{K}^{2} .
$$

Above, the requirement on $\nabla \cdot \boldsymbol{v}$ to be a piecewise $p$-degree polynomial is only employed for clarity of presentation; the genal case is treated in [19, Theorem 3.3] upon addition of appropriate $h p$-data oscillation terms of $\nabla \cdot \boldsymbol{v}$. Note that, importantly, the minimization terms in the right-hand side of (3.2) are completely local in each tetrahedron and do not involve any constraint on the divergence in $K$ nor on the normal component value on faces in $\partial K \cap \overline{\Gamma_{\mathrm{N}}}$. Thus, the approximation by normal-component-continuous piecewise polynomials is comparable to that by discontinuous piecewise polynomials whenever the target function has $\boldsymbol{H}(\operatorname{div}, \Omega)$-regularity, and, moreover, the divergence constraint can be relaxed.

\subsection{Other best-approximations in $H^{1}$ and $\boldsymbol{H}$ (div)}

We will derive below two equivalence results in $\boldsymbol{H}(\operatorname{curl}, \Omega)$ : in $\boldsymbol{L}^{2}(\Omega)$ for the differential operator and in $\boldsymbol{L}^{2}(\Omega)$ under a constraint on the differential operator. The following remarks clarify such complementary possible best-approximations in $H^{1}(\Omega)$ and $\boldsymbol{H}(\operatorname{div}, \Omega)$.

Remark 3.3 (Equivalence of local-best and global-best approximations in $H^{1}, L^{2}$ norm under under the constraint on the gradient). In $H^{1}(\Omega)$, Proposition 3.1 only presents the result in the $H^{1}$ seminorm, i.e., in $\boldsymbol{L}^{2}(\Omega)$ for the differential operator. The other form is, for $v \in H_{0, \mathrm{D}}^{1}(\Omega)$,

$$
\min _{\substack{v_{h} \in \mathcal{P}_{p}\left(\mathcal{T}_{h}\right) \cap H_{0, \mathrm{D}}^{1}(\Omega) \\ \nabla v_{h}=\Pi_{p} \nabla v}}\left\|v-v_{h}\right\|^{2}
$$


where $\boldsymbol{\Pi}_{p}$ is the $\boldsymbol{L}^{2}(\Omega)$-orthogonal projector onto $\nabla\left(\mathcal{P}_{p}\left(\mathcal{T}_{h}\right) \cap H_{0, \mathrm{D}}^{1}(\Omega)\right)$. This constraint alone, however, already fixes the minimizer unless $\Gamma_{\mathrm{D}}=\emptyset$ (in which case only one degree of freedom is left); a simple one-dimensional example shows that (3.3) is not equivalent to

$$
\sum_{K \in \mathcal{T}_{h}} \min _{\substack{v_{h} \in \mathcal{P}_{p}(K) \\ \nabla v_{h}=\Pi_{p, K}\left(\left.\nabla v\right|_{K}\right)}}\left\|v-v_{h}\right\|_{K}^{2}
$$

where $\boldsymbol{\Pi}_{p, K}$ is the $\boldsymbol{L}^{2}(K)$-orthogonal projector onto $\nabla\left(\mathcal{P}_{p}(K)\right)$, and even less so to

$$
\sum_{K \in \mathcal{T}_{h}} \min _{v_{h} \in \mathcal{P}_{p}(K)}\left\|v-v_{h}\right\|_{K}^{2}
$$

Remark 3.4 (Equivalence of local-best and global-best approximations in $\boldsymbol{H}(\mathrm{div}), L^{2}$ norm of the divergence). In $\boldsymbol{H}(\operatorname{div}, \Omega)$, Proposition 3.2 only presents the result in $\boldsymbol{L}^{2}(\Omega)$ under a constraint on the differential operator (the divergence). The other form localizes trivially, since for any $\boldsymbol{v} \in \boldsymbol{H}_{0, \mathrm{~N}}(\operatorname{div}, \Omega)$,

$$
\begin{aligned}
\min _{\boldsymbol{v}_{h} \in \mathcal{R} \mathcal{T}_{p}\left(\mathcal{T}_{h}\right) \cap \boldsymbol{H}_{0, \mathrm{~N}}(\operatorname{div}, \Omega)}\left\|\nabla \cdot\left(\boldsymbol{v}-\boldsymbol{v}_{h}\right)\right\|^{2} & =\sum_{K \in \mathcal{T}_{h}} \min _{\boldsymbol{v}_{h} \in \mathcal{R} \mathcal{T}_{p}(K)}\left\|\nabla \cdot\left(\boldsymbol{v}-\boldsymbol{v}_{h}\right)\right\|_{K}^{2} \\
& =\sum_{K \in \mathcal{T}_{h}}\left\|\nabla \cdot \boldsymbol{v}-\Pi_{p}(\nabla \cdot \boldsymbol{v})\right\|_{K}^{2},
\end{aligned}
$$

where $\Pi_{p}$ is the $L^{2}(\Omega)$-orthogonal projector onto $\mathcal{P}_{p}\left(\mathcal{T}_{h}\right)$.

\section{Equivalence of local-best and global-best approximations in $\boldsymbol{H}$ (curl)}

We present in this section our two main results.

\subsection{Equivalence in the $\boldsymbol{H}$ (curl)-seminorm}

We first consider $\boldsymbol{H}(\operatorname{curl}, \Omega)$ approximations of the curl in the $\boldsymbol{L}^{2}(\Omega)$-norm without any constraint, in a parallel to Proposition 3.1. Recall the partition of the boundary $\partial \Omega=\overline{\Gamma_{\mathrm{D}}} \cup \overline{\Gamma_{\mathrm{N}}}$, with $\Gamma_{\mathrm{D}}$ and $\Gamma_{\mathrm{N}}$ possibly empty.

Theorem 4.1 (Equivalence of local-best and global-best approximations in $\boldsymbol{H}$ (curl), $\boldsymbol{H}$ (curl) seminorm). Let $p \geq 0$ be fixed. Then there exists a constant only depending on the shape-regularity $\kappa_{\mathcal{T}_{h}}$ of the mesh $\mathcal{T}_{h}$ and the polynomial degree $p$ such that for any $\boldsymbol{v} \in \boldsymbol{H}_{0, \mathrm{~N}}(\operatorname{curl}, \Omega)$,

$$
\min _{\boldsymbol{v}_{h} \in \mathcal{N}_{p}\left(\mathcal{T}_{h}\right) \cap \boldsymbol{H}_{0, \mathrm{~N}}(\operatorname{curl}, \Omega)}\left\|\nabla \times\left(\boldsymbol{v}-\boldsymbol{v}_{h}\right)\right\|^{2} \approx \sum_{K \in \mathcal{T}_{h}} \min _{\boldsymbol{v}_{h} \in \mathcal{N}_{p}(K)}\left\|\nabla \times\left(\boldsymbol{v}-\boldsymbol{v}_{h}\right)\right\|_{K}^{2} \cdot
$$

Proof. There trivially holds

$$
\min _{\boldsymbol{v}_{h} \in \mathcal{N}_{p}\left(\mathcal{T}_{h}\right) \cap \boldsymbol{H}_{0, \mathrm{~N}}(\operatorname{curl}, \Omega)}\left\|\nabla \times\left(\boldsymbol{v}-\boldsymbol{v}_{h}\right)\right\|^{2} \geq \sum_{K \in \mathcal{T}_{h}} \min _{\boldsymbol{v}_{h} \in \mathcal{N}_{p}(K)}\left\|\nabla \times\left(\boldsymbol{v}-\boldsymbol{v}_{h}\right)\right\|_{K}^{2},
$$

since the broken minimization set on the right-hand side is (much) larger. This establishes one inequality.

Let $\boldsymbol{v} \in \boldsymbol{H}_{0, \mathrm{~N}}(\operatorname{curl}, \Omega)$ be fixed. Since the $\nabla \times$ operator maps from the space $\boldsymbol{H}_{0, \mathrm{~N}}(\mathrm{curl}, \Omega)$ onto the space $\left\{\boldsymbol{\varphi} \in \boldsymbol{H}_{0, \mathrm{~N}}(\operatorname{div}, \Omega) ; \nabla \cdot \boldsymbol{\varphi}=0\right\}$, see (1.1) and [24] for general boundary conditions, we can set $\boldsymbol{w}:=\nabla \times \boldsymbol{v}$ and have $\boldsymbol{w} \in \boldsymbol{H}_{0, \mathrm{~N}}(\operatorname{div}, \Omega)$ with $\nabla \cdot \boldsymbol{w}=0$. Since, similarly, $\nabla \times$ maps from $\mathcal{N}_{p}\left(\mathcal{T}_{h}\right) \cap \boldsymbol{H}_{0, \mathrm{~N}}(\operatorname{curl}, \Omega)$ onto the space $\left\{\boldsymbol{\varphi}_{h} \in \mathcal{R} \mathcal{T}_{p}\left(\mathcal{T}_{h}\right) \cap \boldsymbol{H}_{0, \mathrm{~N}}(\operatorname{div}, \Omega) ; \nabla \cdot \boldsymbol{\varphi}_{h}=0\right\}$, see [2] or [7, Section 2.5.6], we see

$$
\min _{\boldsymbol{v}_{h} \in \mathcal{N}_{p}\left(\mathcal{T}_{h}\right) \cap \boldsymbol{H}_{0, \mathrm{~N}}(\operatorname{curl}, \Omega)}\left\|\nabla \times\left(\boldsymbol{v}-\boldsymbol{v}_{h}\right)\right\|^{2}=\min _{\substack{\boldsymbol{w}_{h} \in \mathcal{R} \mathcal{T}_{p}\left(\mathcal{T}_{h}\right) \cap \boldsymbol{H}_{0, \mathrm{~N}}(\operatorname{div}, \Omega) \\ \nabla \cdot \boldsymbol{w}_{h}=0}}\left\|\boldsymbol{w}-\boldsymbol{w}_{h}\right\|^{2}=: A .
$$


Now Proposition 3.2 yields

$$
\begin{aligned}
A & \lesssim \sum_{K \in \mathcal{T}_{h}} \min _{\boldsymbol{w}_{h} \in \mathcal{R} \mathcal{T}_{p}(K)}\left\|\boldsymbol{w}-\boldsymbol{w}_{h}\right\|_{K}^{2} \leq \sum_{K \in \mathcal{T}_{h}} \min _{\substack{\boldsymbol{w}_{h} \in \mathcal{R} \mathcal{T}_{p}(K) \\
\nabla \cdot \boldsymbol{w}_{h}=0}}\left\|\boldsymbol{w}-\boldsymbol{w}_{h}\right\|_{K}^{2} \\
& =\sum_{K \in \mathcal{T}_{h}} \min _{\boldsymbol{v}_{h} \in \mathcal{N}_{p}(K)}\left\|\nabla \times\left(\boldsymbol{v}-\boldsymbol{v}_{h}\right)\right\|_{K}^{2},
\end{aligned}
$$

where the inequality $\leq$ is trivial, since the right-hand side adds an additional constraint, and the equality is obtained as above, since

$$
\nabla \times \mathcal{N}_{p}(K)=\left\{\boldsymbol{v}_{h} \in \mathcal{R} \mathcal{T}_{p}(K) ; \nabla \cdot \boldsymbol{v}_{h}=0\right\},
$$

see, e.g., [7, equation (2.3.62)]. This establishes the other inequality.

\subsection{Equivalence in the $L^{2}$-norm removing the constraint on the curl}

We next consider $\boldsymbol{H}$ (curl, $\Omega$ ) approximations in the $\boldsymbol{L}^{2}(\Omega)$-norm with a constraint on the curl, in a parallel to Proposition 3.2. Recall that $\partial \Omega=\overline{\Gamma_{\mathrm{D}}} \cup \overline{\Gamma_{\mathrm{N}}}$, with $\Gamma_{\mathrm{D}}$ and $\Gamma_{\mathrm{N}}$ possibly empty. Below, we for simplicity of presentation only treat functions $\boldsymbol{v}$ with piecewise polynomial curls, $\boldsymbol{v} \in \boldsymbol{H}_{0, \mathrm{D}}(\mathrm{curl}, \Omega)$ with $\nabla \times \boldsymbol{v} \in$ $\left[\mathcal{P}_{p}\left(\mathcal{T}_{h}\right)\right]^{3}$. Note that since then there exits $\boldsymbol{h}_{h} \in \mathcal{N}_{p}\left(\mathcal{T}_{h}\right) \cap \boldsymbol{H}_{0, \mathrm{D}}(\operatorname{curl}, \Omega)$ such that $\nabla \times \boldsymbol{h}_{h}=\nabla \times \boldsymbol{v}$ (see, e.g., [7, equation (2.5.41)] ) and since $\nabla \times\left(\mathcal{N}_{p}\left(\mathcal{T}_{h}\right) \cap \boldsymbol{H}_{0, \mathrm{D}}(\operatorname{curl}, \Omega)\right)=\left\{\boldsymbol{\varphi}_{h} \in \mathcal{R} \mathcal{T}_{p}\left(\mathcal{T}_{h}\right) \cap \boldsymbol{H}_{0, \mathrm{D}}(\operatorname{div}, \Omega) ; \nabla \cdot \boldsymbol{\varphi}_{h}=0\right\}$, this requirement could be equivalently written as asking

$$
\nabla \times \boldsymbol{v} \in\left\{\boldsymbol{\varphi}_{h} \in \mathcal{R} \mathcal{T}_{p}\left(\mathcal{T}_{h}\right) \cap \boldsymbol{H}_{0, \mathrm{D}}(\operatorname{div}, \Omega) ; \nabla \cdot \boldsymbol{\varphi}_{h}=0\right\}
$$

Theorem 4.2 (Equivalence of local-best and global-best approximations in $\boldsymbol{H}$ (curl), $\boldsymbol{L}^{2}$-norm under the constraint on the curl (removing the constraint)). Let $p \geq 0$ be fixed. Then there exists a constant only depending on the shape-regularity $\kappa_{\mathcal{T}_{h}}$ of the mesh $\mathcal{T}_{h}$ and the polynomial degree $p$ such that for any $\boldsymbol{v} \in$ $\boldsymbol{H}_{0, \mathrm{D}}(\operatorname{curl}, \Omega)$ with $\nabla \times \boldsymbol{v} \in\left[\mathcal{P}_{p}\left(\mathcal{T}_{h}\right)\right]^{3}$,

$$
\min _{\substack{\boldsymbol{v}_{h} \in \mathcal{N}_{p}\left(\mathcal{T}_{h}\right) \cap \boldsymbol{H}_{0, \mathrm{D}}(\operatorname{curl}, \Omega) \\ \nabla \times \boldsymbol{v}_{h}=\nabla \times \boldsymbol{v}}}\left\|\boldsymbol{v}-\boldsymbol{v}_{h}\right\|^{2} \approx \sum_{K \in \mathcal{T}_{h}} \min _{\boldsymbol{v}_{h} \in \mathcal{N}_{p}(K)}\left\|\boldsymbol{v}-\boldsymbol{v}_{h}\right\|_{K}^{2} \cdot
$$

Proof. There trivially holds

$$
\min _{\substack{\boldsymbol{v}_{h} \in \mathcal{N}_{p}\left(\mathcal{T}_{h}\right) \cap \boldsymbol{H}_{\mathrm{O}, \mathrm{D}}(\operatorname{curl}, \Omega) \\ \nabla \times \boldsymbol{v}_{h}=\nabla \times \boldsymbol{v}}}\left\|\boldsymbol{v}-\boldsymbol{v}_{h}\right\|^{2} \geq \sum_{K \in \mathcal{T}_{h}} \min _{\boldsymbol{v}_{h} \in \mathcal{N}_{p}(K)}\left\|\boldsymbol{v}-\boldsymbol{v}_{h}\right\|_{K}^{2},
$$

since the broken minimization set on the right-hand side is (much) larger. This establishes one inequality.

Fix $\boldsymbol{v} \in \boldsymbol{H}_{0, \mathrm{D}}(\operatorname{curl}, \Omega)$ with $\nabla \times \boldsymbol{v} \in\left[\mathcal{P}_{p}\left(\mathcal{T}_{h}\right)\right]^{3}$. Choose any $\boldsymbol{h}_{h} \in \mathcal{N}_{p}\left(\mathcal{T}_{h}\right) \cap \boldsymbol{H}_{0, \mathrm{D}}(\operatorname{curl}, \Omega)$ such that $\nabla \times \boldsymbol{h}_{h}=\nabla \times \boldsymbol{v}$. Then $\boldsymbol{v}-\boldsymbol{h}_{h} \in \boldsymbol{H}_{0, \mathrm{D}}(\operatorname{curl}, \Omega)$ is such that $\nabla \times\left(\boldsymbol{v}-\boldsymbol{h}_{h}\right)=\mathbf{0}$, so that there exists (a unique, up to a constant when $\left.\Gamma_{\mathrm{D}}=\emptyset\right) q \in H_{0, \mathrm{D}}^{1}(\Omega)$ such that $\nabla q=\boldsymbol{v}-\boldsymbol{h}_{h}$, see (1.1) and [24] for general boundary conditions. Similarly, for any $\boldsymbol{v}_{h} \in \mathcal{N}_{p}\left(\mathcal{T}_{h}\right) \cap \boldsymbol{H}_{0, \mathrm{D}}(\operatorname{curl}, \Omega)$ such that $\nabla \times \boldsymbol{v}_{h}=\nabla \times \boldsymbol{v}$, $\boldsymbol{v}_{h}-\boldsymbol{h}_{h} \in \mathcal{N}_{p}\left(\mathcal{T}_{h}\right) \cap \boldsymbol{H}_{0, \mathrm{D}}(\operatorname{curl}, \Omega)$ is such that $\nabla \times\left(\boldsymbol{v}_{h}-\boldsymbol{h}_{h}\right)=0$, so that there exists (a unique, up to a constant when $\left.\Gamma_{\mathrm{D}}=\emptyset\right) q_{h} \in \mathcal{P}_{p+1}\left(\mathcal{T}_{h}\right) \cap H_{0, \mathrm{D}}^{1}(\Omega)$ such that $\nabla q_{h}=\boldsymbol{v}_{h}-\boldsymbol{h}_{h}$, see [2] or [7, Section 2.5.6]. Consequently,

$$
\min _{\substack{\boldsymbol{v}_{h} \in \mathcal{N}_{p}\left(\mathcal{T}_{h}\right) \cap \boldsymbol{H}_{0, \mathrm{D}}(\operatorname{curl}, \Omega) \\ \nabla \times \boldsymbol{v}_{h}=\nabla \times \boldsymbol{v}}}\left\|\boldsymbol{v}-\boldsymbol{v}_{h}\right\|^{2}=\min _{v_{h} \in \mathcal{P}_{p+1}\left(\mathcal{T}_{h}\right) \cap H_{0, \mathrm{D}}^{1}(\Omega)}\left\|\nabla\left(q-v_{h}\right)\right\|^{2}=: A .
$$

Now, using the localization of Proposition 3.1, we infer

$$
A \lesssim \sum_{K \in \mathcal{T}_{h}} \min _{v_{h} \in \mathcal{P}_{p+1}(K)}\left\|\nabla\left(q-v_{h}\right)\right\|_{K}^{2}=\sum_{K \in \mathcal{T}_{h}} \min _{\substack{\boldsymbol{v}_{h} \in \mathcal{N}_{p}(K) \\ \nabla \times \boldsymbol{v}_{h}=\nabla \times \boldsymbol{v}}}\left\|\boldsymbol{v}-\boldsymbol{v}_{h}\right\|_{K}^{2},
$$

where, in the equality, we have used the fact that for any $v_{h} \in \mathcal{P}_{p+1}(K)$, there exists a unique $\boldsymbol{v}_{h} \in \mathcal{N}_{p}(K)$ with $\nabla \times \boldsymbol{v}_{h}=\left.(\nabla \times \boldsymbol{v})\right|_{K}$ such that $\nabla v_{h}=\boldsymbol{v}_{h}-\boldsymbol{h}_{h}$ (following [7, equation (2.3.56)], $\boldsymbol{v}_{h}=\nabla v_{h}+\boldsymbol{h}_{h}$ ). Finally, employing Lemma A.1 below which crucially allows to remove the curl constraint establishes the other inequality. 


\section{A Equivalence of constrained and unconstrained best-approxima- tion in $\boldsymbol{H}$ (curl) on a tetrahedron}

The following important lemma follows along the lines of [19, Lemma A.1], on the basis of the breakthrough result of Costabel and McIntosh in [16, Proposition 4.2]. Interestingly enough, the constant hidden in the inequality is here independent of the polynomial degree $p$.

Lemma A.1 (Equivalence of constrained and unconstrained best-approximation on a tetrahedron). Let a polynomial degree $p \geq 0$ and a tetrahedron $K$ be fixed. Then there exists a constant only depending on the shape-regularity $\kappa_{K}:=h_{K} / \rho_{K}$ of $K$ such that for any $\boldsymbol{v} \in \boldsymbol{H}(\operatorname{curl}, K)$ with $\nabla \times \boldsymbol{v} \in\left[\mathcal{P}_{p}(K)\right]^{3}$,

$$
\min _{\boldsymbol{v}_{h} \in \mathcal{N}_{p}(K)}\left\|\boldsymbol{v}-\boldsymbol{v}_{h}\right\|_{K} \leq \min _{\substack{\boldsymbol{v}_{h} \in \mathcal{N}_{p}(K) \\ \nabla \times \boldsymbol{v}_{h}=\nabla \times \boldsymbol{v}}}\left\|\boldsymbol{v}-\boldsymbol{v}_{h}\right\|_{K} \lesssim \min _{\boldsymbol{v}_{h} \in \mathcal{N}_{p}(K)}\left\|\boldsymbol{v}-\boldsymbol{v}_{h}\right\|_{K}
$$

Proof. The first inequality in (A.1) is trivial, since the right-hand side adds an additional constraint. So we focus on the second one. Denote respectively

$$
\begin{gathered}
\boldsymbol{\tau}_{h}:=\arg \min _{\substack{\boldsymbol{v}_{h} \in \mathcal{N}_{p}(K) \\
\nabla \times \boldsymbol{v}_{h}=\nabla \times \boldsymbol{v}}}\left\|\boldsymbol{v}-\boldsymbol{v}_{h}\right\|_{K}, \\
\widetilde{\boldsymbol{\tau}}_{h}:=\arg \min _{\boldsymbol{v}_{h} \in \mathcal{N}_{p}(K)}\left\|\boldsymbol{v}-\boldsymbol{v}_{h}\right\|_{K}
\end{gathered}
$$

the constrained and unconstrained minimizers. We then need to show

$$
\left\|\boldsymbol{v}-\boldsymbol{\tau}_{h}\right\|_{K} \lesssim\left\|\boldsymbol{v}-\widetilde{\boldsymbol{\tau}}_{h}\right\|_{K}
$$

Using (4.2)-(4.3), on the element $K, \nabla \times\left(\boldsymbol{v}-\widetilde{\boldsymbol{\tau}}_{h}\right) \in\left\{\boldsymbol{v}_{h} \in \mathcal{R} \mathcal{T}_{p}(K) ; \nabla \cdot \boldsymbol{v}_{h}=0\right\}$. Thus, we can use [16, Proposition 4.2], cf. also the reformulation in [11, Theorem 2], stipulating the existence of $\boldsymbol{w}_{h} \in \mathcal{N}_{p}(K)$ with $\nabla \times \boldsymbol{w}_{h}=\nabla \times\left(\boldsymbol{v}-\widetilde{\boldsymbol{\tau}}_{h}\right)$ such that

$$
\left\|\boldsymbol{w}_{h}\right\|_{K} \lesssim \min _{\substack{\boldsymbol{\varphi} \in \boldsymbol{H}(\operatorname{curl}, K) \\ \nabla \times \boldsymbol{\varphi}=\nabla \times\left(\boldsymbol{v}-\widetilde{\boldsymbol{\tau}}_{h}\right)}}\|\boldsymbol{\varphi}\|_{K}
$$

Shifting now the right-hand side of $(\mathrm{A} .4)$ by $\widetilde{\tau}_{h}$, we arrive at

$$
\left\|\boldsymbol{w}_{h}\right\|_{K} \lesssim \min _{\substack{\boldsymbol{\varphi} \in \boldsymbol{H}(\operatorname{curl}, K) \\ \nabla \times \boldsymbol{\varphi}=\nabla \times \boldsymbol{v}}}\left\|\boldsymbol{\varphi}-\widetilde{\boldsymbol{\tau}}_{h}\right\|_{K} \leq\left\|\boldsymbol{v}-\widetilde{\boldsymbol{\tau}}_{h}\right\|_{K}
$$

where the inequality $\leq$ is trivial, since $\boldsymbol{v} \in \boldsymbol{H}(\operatorname{curl}, K)$ with $\nabla \times \boldsymbol{v}=\nabla \times \boldsymbol{v}$. Note that $\left(\boldsymbol{w}_{h}+\widetilde{\boldsymbol{\tau}}_{h}\right) \in \mathcal{N}_{p}(K)$ with $\nabla \times\left(\boldsymbol{w}_{h}+\widetilde{\boldsymbol{\tau}}_{h}\right)=\bar{\nabla} \times \boldsymbol{v}$. Thus, $\boldsymbol{w}_{h}+\widetilde{\boldsymbol{\tau}}_{h}$ belongs to the minimization set in (A.2a), and the minimization property (A.2a) of $\boldsymbol{\tau}_{h}$ implies $\left\|\boldsymbol{v}-\boldsymbol{\tau}_{h}\right\|_{K} \leq\left\|\boldsymbol{v}-\left(\boldsymbol{w}_{h}+\widetilde{\boldsymbol{\tau}}_{h}\right)\right\|_{K}$. Thus, by virtue of the triangle inequality and using (A.5), we altogether infer

$$
\left\|\boldsymbol{v}-\boldsymbol{\tau}_{h}\right\|_{K} \leq\left\|\boldsymbol{v}-\left(\boldsymbol{w}_{h}+\widetilde{\boldsymbol{\tau}}_{h}\right)\right\|_{K} \leq\left\|\boldsymbol{v}-\widetilde{\boldsymbol{\tau}}_{h}\right\|_{K}+\left\|\boldsymbol{w}_{h}\right\|_{K} \lesssim\left\|\boldsymbol{v}-\widetilde{\boldsymbol{\tau}}_{h}\right\|_{K}
$$

i.e., (A.3), and the proof is finished.

\section{References}

[1] Adams, R. A. Pure and Applied Mathematics, Vol. 65. Sobolev spaces. Academic Press [A subsidiary of Harcourt Brace Jovanovich, Publishers], New York-London, 1975.

[2] Arnold, D. N., Falk, R. S., and Winther, R. Finite element exterior calculus, homological techniques, and applications. Acta Numer. 15 (2006), 1-155. https://doi.org/10.1017/S0962492906210018.

[3] Aurada, M., Feischl, M., Kemetmüller, J., Page, M., and Praetorius, D. Each $H^{1 / 2}$-stable projection yields convergence and quasi-optimality of adaptive FEM with inhomogeneous Dirichlet data in $\mathbb{R}^{d}$. ESAIM Math. Model. Numer. Anal. 47 (2013), 1207-1235. https://doi.org/10.1051/m2an/2013069. 
[4] Bank, R. E., and Ovall, J. S. Some remarks on interpolation and best approximation. Numer. Math. 137 (2017), 289-302. https://doi.org/10.1007/s00211-017-0877-7.

[5] Bank, R. E., and Yserentant, H. A note on interpolation, best approximation, and the saturation property. Numer. Math. 131 (2015), 199-203. https://doi.org/10.1007/s00211-014-0687-0.

[6] Bernardi, C., and Hecht, F. Quelques propriétés d'approximation des éléments finis de Nédélec, application à l'analyse a posteriori. C. R. Math. Acad. Sci. Paris 344 (2007), 461-466. https: //doi.org/10.1016/j.crma.2007.02.010.

[7] Boffi, D., Brezzi, F., and Fortin, M. Mixed finite element methods and applications, vol. 44 of Springer Series in Computational Mathematics. Springer, Heidelberg, 2013. https://doi.org/10. 1007/978-3-642-36519-5.

[8] Canuto, C., Nochetto, R. H., Stevenson, R. P., and Verani, M. Convergence and optimality of $h p-$ AFEM. Numer. Math. 135 (2017), 1073-1119. https://doi.org/10.1007/s00211-016-0826-x.

[9] Carstensen, C., Peterseim, D., and Schedensack, M. Comparison results of finite element methods for the Poisson model problem. SIAM J. Numer. Anal. 50 (2012), 2803-2823. https://doi.org/10. $1137 / 110845707$.

[10] Carstensen, C., and Schedensack, M. Medius analysis and comparison results for first-order finite element methods in linear elasticity. IMA J. Numer. Anal. 35 (2015), 1591-1621. https://doi.org/ 10.1093/imanum/dru048.

[11] Chaumont-Frelet, T., Ern, A., and Vohralík, M. Polynomial-degree-robust $\boldsymbol{H}$ (curl)-stability of discrete minimization in a tetrahedron. C. R. Math. Acad. Sci. Paris 358 (2020), 1101-1110. https://doi. org/10.5802/crmath. 133 .

[12] Chaumont-Frelet, T., Nicaise, S., and Pardo, D. Finite element approximation of electromagnetic fields using nonfitting meshes for geophysics. SIAM J. Numer. Anal. 56 (2018), 2288-2321. https: //doi.org/10.1137/16M1105566.

[13] Christiansen, S. H., and Winther, R. Smoothed projections in finite element exterior calculus. Math. Comp. 77 (2008), 813-829. http://dx.doi.org/10.1090/S0025-5718-07-02081-9.

[14] Ciarlet, Jr., P. On the approximation of electromagnetic fields by edge finite elements. Part 1: Sharp interpolation results for low-regularity fields. Comput. Math. Appl. 71 (2016), 85-104. https://doi. org/10.1016/j. camwa.2015.10.020.

[15] Clément, P. Approximation by finite element functions using local regularization. RAIRO Anal. Numer. 9 (1975), $77-84$.

[16] Costabel, M., and McIntosh, A. On Bogovskiı̌ and regularized Poincaré integral operators for de Rham complexes on Lipschitz domains. Math. Z. 265 (2010), 297-320. http://dx.doi.org/10.1007/ s00209-009-0517-8.

[17] Demkowicz, L. Polynomial exact sequences and projection-based interpolation with application to Maxwell equations. In Mixed finite elements, compatibility conditions, and applications, D. Boffi, F. Brezzi, L. F. Demkowicz, R. G. Durán, R. S. Falk, and M. Fortin, Eds., vol. 1939 of Lecture Notes in Mathematics. Springer-Verlag, Berlin; Fondazione C.I.M.E., Florence, 2008, pp. 101-158. Lectures given at the C.I.M.E. Summer School held in Cetraro, June 26-July 1, 2006, Edited by D. Boffi and L. Gastaldi, https://doi.org/10.1007/978-3-540-78319-0_3.

[18] Demkowicz, L., and Buffa, A. $H^{1}, H$ (curl) and $H$ (div)-conforming projection-based interpolation in three dimensions. Quasi-optimal p-interpolation estimates. Comput. Methods Appl. Mech. Engrg. 194 (2005), 267-296. https://doi.org/10.1016/j.cma.2004.07.007.

[19] Ern, A., Gudi, T., Smears, I., and Vohralík, M. Equivalence of local- and global-best approximations, a simple stable local commuting projector, and optimal $h p$ approximation estimates in $\boldsymbol{H}(\mathrm{div})$. IMA J. Numer. Anal. (2021). DOI 10.1093/imanum/draa103, http://dx.doi.org/10.1093/imanum/ draa103. 
[20] Ern, A., and Guermond, J.-L. Mollification in strongly Lipschitz domains with application to continuous and discrete de Rham complexes. Comput. Methods Appl. Math. 16 (2016), 51-75. https://doi.org/ 10.1515/cmam-2015-0034.

[21] Ern, A., and Guermond, J.-L. Finite element quasi-interpolation and best approximation. ESAIM Math. Model. Numer. Anal. 51 (2017), 1367-1385. https://doi.org/10.1051/m2an/2016066.

[22] Ern, A., and Guermond, J.-L. Analysis of the edge finite element approximation of the Maxwell equations with low regularity solutions. Comput. Math. Appl. 75 (2018), 918-932. https://doi.org/ $10.1016 / j$. camwa. 2017.10 .017 .

[23] Falk, R. S., and Winther, R. Local bounded cochain projections. Math. Comp. 83 (2014), 2631-2656. http://dx.doi.org/10.1090/S0025-5718-2014-02827-5.

[24] Fernandes, P., and Gilardi, G. Magnetostatic and electrostatic problems in inhomogeneous anisotropic media with irregular boundary and mixed boundary conditions. Math. Models Methods Appl. Sci. 7 (1997), 957-991. https://doi.org/10.1142/S0218202597000487.

[25] Girault, V., and Raviart, P.-A. Theory and algorithms. Finite element methods for Navier-Stokes equations, vol. 5 of Springer Series in Computational Mathematics. Springer-Verlag, Berlin, 1986.

[26] Licht, M. W. Smoothed projections and mixed boundary conditions. Math. Comp. 88 (2019), 607-635. https://doi.org/10.1090/mcom/3330.

[27] Melenk, J. M., and Rojik, C. On commuting $p$-version projection-based interpolation on tetrahedra. Math. Comp. 89 (2020), 45-87. https://doi.org/10.1090/mcom/3454.

[28] Monk, P. Finite element methods for Maxwell's equations. Numerical Mathematics and Scientific Computation. Oxford University Press, New York, 2003. https://doi.org/10.1093/acprof:oso/ 9780198508885.001 .0001$.

[29] Nédélec, J.-C. Mixed finite elements in $\mathbb{R}^{3}$. Numer. Math. 35 (1980), 315-341.

[30] Raviart, P.-A., and Thomas, J.-M. A mixed finite element method for 2nd order elliptic problems. In Mathematical aspects of finite element methods (Proc. Conf., Consiglio Naz. delle Ricerche (C.N.R.), Rome, 1975). Springer, Berlin, 1977, pp. 292-315. Lecture Notes in Math., Vol. 606.

[31] Schöberl, J. Commuting quasi-interpolation operators for mixed finite elements. Tech. rep. ISC-01-10MATH, Texas A\&M University, https://isc.tamu.edu/resources/preprints/2001/2001-10.pdf, 2001.

[32] Schöberl, J. A multilevel decomposition result in $\boldsymbol{H}$ (curl). In Multigrid, multilevel and multiscale methods, Proceedings of the $8^{\text {th }}$ European Multigrid Conference (2005), P. Wesseling, C. Oosterlee, and P. Hemker, Eds., TU Delft.

[33] Scott, L. R., and Zhang, S. Finite element interpolation of nonsmooth functions satisfying boundary conditions. Math. Comp. 54 (1990), 483-493. https://doi.org/10.2307/2008497.

[34] Tantardini, F., Veeser, A., and Verfürth, R. Robust localization of the best error with finite elements in the reaction-diffusion norm. Constr. Approx. 42 (2015), 313-347. https://doi.org/10.1007/ s00365-015-9291-5.

[35] Veeser, A. Approximating gradients with continuous piecewise polynomial functions. Found. Comput. Math. 16 (2016), 723-750. http://dx.doi.org/10.1007/s10208-015-9262-z.

[36] Veeser, A. Best error localizations for piecewise polynomial approximation of gradients, functions and functionals. In Numerical mathematics and advanced applications-ENUMATH 2017, vol. 126 of Lect. Notes Comput. Sci. Eng. Springer, Cham, 2019, pp. 357-365. https://doi.org/10.1007/ 978-3-319-96415-7_31. 